\title{
Treating Intellectual Disability; Look for Creatine Peaks in the Brain
}

Can. J. Neurol. Sci. 2011; 38: 669-670

Intellectual disability (ID) is a heavy burden both on an individual and society, as its effects last a lifetime. Impairments due to cognitive subnormality (IQ<70) carry social, psychological and economic consequences. A prevalence rate of $3-4 / 1000$ individuals is estimated, and in a majority of cases no definite cause is identifiable'. Genetic factors ( chromosomal aberrations, microdeletions, single gene defects together) account for $25-40 \%$ of ID and the majority are not treatable even if diagnosis is made early ${ }^{2}$. However, single gene disorders categorized under the rubric of inborn errors of metabolism offer unique opportunities where early diagnosis and institution of specific therapy can go a long way to improve neurological outcomes for affected individuals ${ }^{3}$. The first and perhaps most striking success for such interventions was made with phenylketonuria, where the reduction of dietary phenylalanine made a significant impact on the outcome of a disorder that was once considered a major cause of severe ID and neurological handicap. Since that initial step towards recognition of treatable/preventable causes, it has been estimated that there are more than 70 diagnosable conditions that are considered as potentially treatable. One group of disorders, the "cerebral creatine deficiency syndromes (CCDS)" are gaining increasing prominence.

In this issue of the Journal, Hinnell et al bring to our attention the condition of guanidinoacetate methyltransferase (GAMT) deficiency diagnosed in adulthood in two siblings where the diagnosis was made after extensive investigations ${ }^{4}$. In retrospect, they point out in their discussion, that biochemical clues to this condition had been missed. The condition could have also been diagnosed earlier by using the non-invasive technique of magnetic resonance spectroscopy.

The first report of a patient with CCDS was described by Sylvia Stockler and colleagues then at the University of Gottingen in $1994^{5}$. In that paper, the authors made several important observations; 1 ) cerebral depletion of creatine was responsible for the symptoms of a hitherto described neurological condition manifesting with extrapyramidal symptoms, 2) creatine depletion in the brain could be easily identified using magnetic resonance spectroscopy, a noninvasive methodology, and 3) the condition could be corrected with arginine restriction, supplementation of ornithine and creatine monohydrate.

Since that initial description, three distinct biochemical disorders caused by mutations in genes that encode enzymes of creatine biosynthesis and transport have been identified. These are guanidinoacetate methyltransferase deficiency, L-arginineglycine amidinotransferase (AGAT), and creatine transporter (SLC6A8 deficiency) ${ }^{6}$. It has been shown that CCDS can account for a prevalence of nearly $1.9 \%$ of sporadic cases of mental retardation in boys and an even higher proportion in familial cases ${ }^{7}$. The greater impact on males is accounted for by location of the creatine transporter gene is on the $\mathrm{X}$ chromosome. Each of these three conditions can be associated with intellectual disability and a highly variable neurological phenotype associated with epileptic seizures, neurobehavioral symptoms of autism, and movement disorders. The common denominator for each of the three disorders is cerebral creatine depletion. Creatine depletion in the brain can be identified using the technique of magnetic resonance spectroscopy which only takes a few minutes of additional time in routine clinical magnetic resonance imaging (MRI) scanners now available in most major medical centres ${ }^{8}$. The spectral tracing obtained from brain volumes ranging from $1-8 \mathrm{cc}$ gives enough information to quantify several cerebral metabolites including; creatine $(\mathrm{Cr})$, choline (Ch), and $\mathrm{N}$-acetyl aspartate (NAA), lactate, myoinositol, and glucose 9 . The absence of a creatine peak in the presence of normal NAA and Ch peaks is a considered sine qua non of CCDS. Females with SLC6A8 deficiency are the exception as they show only a partial depletion of the cerebral creatine pool. ${ }^{8,10}$

Biochemical confirmation begins with the estimation of guanidine acetate, creatine and creatinine in blood and urine. Low plasma creatinine estimated by the appropriate quantitative methods also serves as a useful biochemical marker ${ }^{11}$. However assays of creatinine in 24 hour urine samples may be misleading in young children and infants with low muscle mass, factors that may be reflected in low values ${ }^{10}$. Hinnell et al further point out that the assay of creatinine in the serum should be carried out by high performance liquid chromatography or stable isotope dilution liquid chromatography electrospray mass spectrometry (ID LC-MSMS), as conventional laboratory methods may over estimate creatinine values when they are at low levels giving rise to spuriously normal values ${ }^{4}$.

Molecular diagnostic testing is currently available for each of the three disorders. Of the three conditions, creatine transporter deficiency is $\mathrm{X}$ linked recessive, while the other two are inherited as autosomal recessive. Treatment for GAMT consists of arginine restriction, creatine monohydrate and ornithine supplementation $^{6,12}$.

Published clinical data suggest that even when the diagnosis is delayed, considerable improvements are seen in clinical symptom control of seizures and behavior. However intellectual disability does not appear to consistently improve in all patients in whom the diagnosis was delayed ${ }^{13}$. In AGAT deficiency, again, creatine supplementation leads to improvements in severity of clinical symptoms. Whether all of the manifestations of CCDS are preventable following early diagnosis and treatment is still under investigation ${ }^{10}$ Symptoms in creatine transporter deficiency have proven most resistant to treatment for obvious reasons ${ }^{8}$. In the absence of the creatine transporter, creatine is unable to get past the blood brain barrier. However it is heartening to note that in a female patient with SLC6A8 
deficiency and intractable epilepsy, supplementation with creatine monohydrate, L-arginine and L-glycine resulted in the complete resolution of seizures ${ }^{14}$.

The paper by Hinnell et al, draws our attention to the phenotypic variability in presentations of CCDS, further emphasizing the need for clinicians to consider this group of disorders in the differential diagnosis of patients with intellectual disability and neurological handicap ${ }^{4}$. Clearly timely diagnosis and potential treatment options that become available once diagnosis is established, promise substantial improvements in the quality of life for affected individuals with CCDS. Thus, clinicians (pediatricians neurologists), biochemical geneticists, and neuroradiologists need to recognize the value of incorporating MRS techniques in the investigation of every patient who undergoes an evaluation for intellectual disability (with or without additional neurological symptoms) so that cerebral creatine depletion does not go unrecognized ${ }^{15}$.

\section{ACKNOWLEDGEMENTS}

The author thanks Dr. C. Prasad, Biochemical Genetics, Genetics Program of South Western Ontario and Dr. C.A. Rupar, Director of the Biochemical Genetics Laboratory, Department of Pediatrics Faculty of Medicine, University of Western Ontario, London, Ontario, Canada, for discussion and their valuable constructive comments in the writing of this editorial.

Asuri N. Prasad Schulich School of Medicine and Dentistry University of Western Ontario, London, Ontario, Canada

\section{REFERENCES}

1. McLaren J, Bryson SE. Review of recent epidemiological studies of mental retardation: prevalence, associated disorders, and etiology. Am J Ment Retard. 1987;92:243-54.

2. Rauch A, Hoyer J, Guth S, et al. Diagnostic yield of various genetic approaches in patients with unexplained developmental delay or mental retardation. Am J Med Genet A. 2006;140:2063-74.

3. Levy HL. Nutritional therapy for selected inborn errors of metabolism. J Am Coll Nutr. 1989;8 Suppl:54S-60.

4. Hinnell C, Samuel M, Alkufri F, et al. Creatine deficiency syndromes: diagnostic pearls and pitfalls. Can J Neurol Sci. 2011;38(5):765-7.

5. Stockler S, Holzbach U, Hanefeld F, et al. Creatine deficiency in the brain: a new, treatable inborn error of metabolism. Pediatr Res. 1994;36:409-13.

6. Schulze A. Creatine deficiency syndromes. Mol Cell Biochem. 2003;244:143-50.

7. Lion-Francois L, Cheillan D, Pitelet G, et al. High frequency of creatine deficiency syndromes in patients with unexplained mental retardation. Neurology. 2006;67:1713-14.

8. Stockler S, Schutz PW, Salomons GS. Cerebral creatine deficiency syndromes: clinical aspects, treatment and pathophysiology. Subcell Biochem. 2007;46:149-66.

9. Novotny E, Ashwal S, Shevell M. Proton magnetic resonance spectroscopy: an emerging technology in pediatric neurology research. Pediatr Res. 1998;44:1-10.

10. Mercimek-Mahmutoglu S, Stockler-Ipsiroglu S. Creatine deficiency syndromes. In: Pagon RA, Bird TD, Dolan CR, Stephens K, editors. Gene reviews. Seattle (WA): 1993.

11. Bodamer OA, Iqbal F, Muhl A, et al. Low creatinine: the diagnostic clue for a treatable neurologic disorder. Neurology. 2009;72: 854-5.

12. Schulze A, Battini R. Pre-symptomatic treatment of creatine biosynthesis defects. Subcell Biochem. 2007;46:167-81.

13. Mercimek-Mahmutoglu S, Stoeckler-Ipsiroglu $S$, Adami A, et al. GAMT deficiency: features, treatment, and outcome in an inborn error of creatine synthesis. Neurology. 2006;67:480-4.

14. Mercimek-Mahmutoglu S, Connolly MB, Poskitt KJ, et al. Treatment of intractable epilepsy in a female with SLC6A8 deficiency. Mol Genet Metab. 2010;101:409-12.

15. Ross BD, Kaye EM. Creatin' news. Neurology. 1999;53:249-50. 